\title{
New pattern recognition methods for identifying oil spills from satellite remote sensing data
}

\author{
Fahad Alawadi ${ }^{* a}$ \\ ${ }^{a}$ School of Ocean and Earth Science, University of Southampton, Waterfront Campus, Empress \\ Dock, Southampton, S014 3ZH, UK
}

\begin{abstract}
The early detection and identification of oil spills are critical prerequisites for performing cost-effective maritime salvage operations.

This paper presents a new approach for distinguishing oil spills that are produced by stationary offshore sources, during their early phase of occurrence. The results were reached after analyzing over 100 images of satellite remote sensing data that were produced by either active microwave sensors like the Synthetic Aperture Radar (SAR) sensors or passive optical sensors like the Moderate Resolution Imaging Spectroradiometer (MODIS).

The laws of conservation of mass and momentum that describe the dynamics of an oil spill over the water surface, were used for the development of a new detection algorithm that encompasses a parallel concept of shape conservation. The validity of this new empirical algorithm depends upon a number of assumptions that were made about the oil viscosity, temperature, water currents, wind speeds and the spills' spatial extent and duration.

It can also be shown that unique texture differences can be revealed between an oil spill and other look-alikes' features like, for example, wind patterns, rain cells and algal mats by applying edge filtering operations on the patches that are under investigation, and therefore the reduction of false positives.

The work presented here may have profound implications on future studies that examine the use of automatic recognitions methods, that are based on pattern and texture analysis. The results may also lead to new methodologies by which the dispersion and trajectory models of oil spills can be studied in new detail and ultimately used in environmental impact assessment operations.
\end{abstract}

Keyword List: MODIS, Oil spill, Oil spread index, SAR, Texture, Pattern recognition, Edge filtering

fa2x07@soton.ac.uk; fahad@ropme.org

Image and Signal Processing for Remote Sensing XV, edited by Lorenzo Bruzzone, Claudia Notarnicola, Francesco Posa, Proc. of SPIE Vol. 7477, 74770X · @ 2009 SPIE · CCC code: 0277-786X/09/\$18 · doi: 10.1117/12.828845 


\section{INTRODUCTION}

A variety of satellite remote sensing sensors are used for the detection of oil spills, amongst which, the Synthetic Aperture Radar (SAR) sensor remains the most popular. For wind speeds that fall within this range $(2-3 \mathrm{~m} / \mathrm{s}<$ wind $<$ $12-15 \mathrm{~m} / \mathrm{s}$ ), oil spills appear as dark patches in the SAR data because they dampen the capillary and short gravity waves and hence reduce the Bragg's backscattering. [26].

Sources other than oil spills, may produce similar dark patterns giving rise to misinterpretation and false positives. This oil look-alike phenomena can be attributed to a variety of reasons such as natural surface films (biogenic), low wind speed areas (wind speed $<3 \mathrm{~m} / \mathrm{s}$ ), wind sheltering by land, internal waves, rain cells, cold upwellings, current shear zones and algal mat formations. [7,11].

Passive optical sensors, like the Moderate Resolution Imaging Spectroradiometer (MODIS) are not commonly used for the detection of oil spills due to their signal attenuation during atmospheric conditions such as cloud cover, dust storms and night overpasses [9]. It has nevertheless, proven its success in detecting relatively large oil spills ( 100pixels), especially in regions that are characterised with $\approx 80 \%$ cloud-free conditions [1]. The Regional Organization for the Protection of the Marine Environment Sea Area (RSA) ${ }^{1}$ with its MODIS station as a prime example for the success of MODIS in detecting oil spills. In MODIS oil spills can appear either dark (negative contrast) or bright (positive contrast) depending on their relative position to the sun glint and angle of observation $[4,6,16]$.

Several papers have already detailed the various procedures used in discriminating between oil spills and look-alikes in terms of the shape and texture that each make at the water surface. Although such methods were commonly used on the SAR data, their applicability can be extended to include data produced by optical sensors like MODIS [15]. Table 1 shows some of the most common methods implemented in shape and texture analysis of oil spills.

Different forces and parameters influence the dynamics of oil spills in water. Three stages have been postulated to explain the spread of oil slicks in water [2,8]. In the first stage, the gravitational forces, caused by the density difference between oil and water, will control the spreading of oil across the surface; inertia is the main retarding force at this stage $[12,13,20]$. In the second stage, the inertial forces will become negligible to be dominated instead by a retarding force assumed to be the viscous shear on the bottom of the slick [25]. In the third and final stage, when the slick has become sufficiently thin, the inter-facial (surface tension) forces will dominate to further its spread [10]. This spreading will continue, at least as long as the surface and inter-facial tensions are unchanged by the change in the oil's physical, chemical and biological properties, collectively called weathering.

Weathering depends on a number of factors such as oil viscosity, wave strength and temperature [25]. For example, high frequency conditions, short term wind patterns and turbulent surface currents will greatly enhance the rate of spread and break up the slick in a process called "shear diffusion" [6,23]. Less viscous oils, like lighter refined "fuel oils" will spread at a higher rate than heavy "crude oils", but because viscosity is temperature dependant, in high temperature waters oil viscosity will decrease, and it will therefore make it more susceptible to spread than cold waters [25].

This paper is proposing a new technique to recognise oil spills viewed from the SAR and optical data on the basis of their shape and texture, provided that these spills have resulted from an offshore stationary object and not from a moving source. This method aims to provide environmental scientists with a rapid method to detect such spills with the least available ancillary data, such as wind and current speeds.

RSA is the sea area surrounded by the eight Member States of ROPME: Bahrain, I.R. Iran, Iraq, Kuwait, Oman, Qatar, Saudi Arabia and the United Arab Emirates. RSA is defined as extending between the following geographic latitudes and longitudes, respectively: $16^{\circ} 39^{\prime} \mathrm{N}, 53^{\circ} 3^{\prime} 30^{\prime \prime} \mathrm{E} ; 6^{\circ} 00^{\prime} \mathrm{N}, 53^{\circ} 25^{\prime} \mathrm{E} ; 17^{\circ} 00^{\prime} \mathrm{N}, 56^{\circ} 30^{\prime} \mathrm{E} ; 20^{\circ} 30^{\prime} \mathrm{N}, 60^{\circ} 00^{\prime} \mathrm{E} ; 5^{\circ} 04^{\prime} \mathrm{N}, 61^{\circ} 25^{\prime} \mathrm{E}$ [20]. 


\begin{tabular}{ccc}
\hline No & Features & Code \\
\hline 1 & Area & $\mathrm{A}$ \\
2 & Perimeter & $\mathrm{P}$ \\
3 & Perimeter to area ratio & P/A \\
4 & Complexity & $\mathrm{C}$ \\
5 & Shape factor I & SP1 \\
\hline 6 & Shape factor II & SP2 \\
7 & Object mean value & OMe \\
8 & Object standard deviation & OSd \\
9 & Object power to mean ratio & Opm \\
10 & Background mean value & BMe \\
\hline 11 & Background standard deviation & BSd \\
12 & Background power to mean ratio & Bpm \\
13 & Ratio of the power to mean ratios & Opm/Bpm \\
14 & Mean contrast & ConMe \\
15 & Max contrast & ConMax \\
\hline 16 & Mean contrast ratio & ConRaMe \\
17 & Standard deviation contrast ratio & ConRaSd \\
18 & Local area contrast ratio & ConLa \\
19 & Mean border gradient & GMe \\
20 & Standard deviation border gradient & GSd \\
\hline 21 & Max border gradient & GMax \\
22 & Mean Difference to Neighbors & NDm \\
23 & Spectral texture & TSp \\
24 & Shape texture & TSh \\
25 & Mean Haralick texture & THm \\
\hline
\end{tabular}

Table 1 The most common methods used in oil spill feature extraction processing [24]

\section{METHOD}

The method outlined here is aimed at identifying fresh oil spills that result from offshore stationary sources, such as oil platforms. Spills originating from inshore sources were excluded from this study to eliminate the additional factors that may interfere with the natural spread of the oil, like for example river discharges along the coast lines, baroclinic currents, tidal currents and stokes drift [3].

A number of assumptions were made to maintain the conditions under which oil continues to spread according to the circumstances explained in stage 2 earlier in the introduction, and therefore, the viscosity changes that oil may experience are negligible.

The analysis was intended exclusively for unweathered (relatively fresh) oil spills to exclude the factors that may disrupt the original viscous nature of oil and therefore alter its shape structure. Factors like strong wind and sea currents, extreme temperature variations or the adding of dispersants to oil which may lead to its de-fragmentation.

The image processing analysis has covered more than 100 different SAR images, collected from historically confirmed oil spills which have occurred at different geographic regions of the world. MODIS data was also used as our case study for optical sensors. MODIS is characterised with a large pixel size (maximum resolution is $250 \mathrm{~m} /$ pixel), therefore the 
shape and texture analysis was only possible for relatively large spills (covering a region $>2500$ pixels). Therefore, a spill that may appear scattered or inhomogeneous when viewed from a SAR sensor, may appear integrated as whole when viewed from an optical sensor like MODIS.

Oil spills always appear as dark patches in the data of SAR relative to their surrounding waters because they dampen the capillary and short gravity waves, and therefore reduce the Bragg's backscattering [26]. The contrast between the spill and its surroundings depends on a number of parameters like wind speed, wave height, and the amount of oil spilled [22]. These same conditions are also valid for determining the spills' contrast in MODIS data, in addition to other parameters, like the optical properties of oil, the film's thickness, light absorption and scattering coefficients, sea depth, angle of illumination and the angle of observation $[17,18]$.

\subsection{The slick feature extraction methodology}

The process adopted for feature extraction can be explained thus: apply the sobel filter on the SAR image to highlight the edges of the suspected dark patch. Before applying the sobel operator on the MODIS image and after excluding the clouds from the scene, we construct a grey scale image using the NIR $(869 \mathrm{~nm})$ band available in the L1B 250m data by using the band relation shown in equation 1.

$$
\frac{1}{\left(B_{2}\right)^{2}}
$$

where $B_{2}$ is the radiance at $869 \mathrm{~nm}^{2}$.

The purpose for this image was to inverses the contrast of the scene and improve the discrimination between the surface water objects (such as algal mats and oil spills) from the dark water background.

The spill's edge features will then be assessed in terms of: (a) uniformity of edge brightness; (b) continuity; and (c) texture granularity or complexity. By continuity we mean that it is free from sharp edges ( $\mathrm{C}_{0}$ curvature type) or geometric discontinuities (Figure 1), except at the source of the spill. Uniform edge brightness indicates that the oil spill is either fresh or of high viscosity type. Semi diluted or discontinuous edges may on the other hand indicate that the oil has already become weathered oil, or less viscous oil type, or it is of low concentration (sheen). Further texture analysis is carried out by performing additional contrast stretching operation to highlight the granularity structures on the patch's surface. An oil spill area should appear similar but less in complexity and granularity than the surrounding waters, since the viscous oil area stops the small wave generation and propagation, which Figure 2 highlights. In the final stage, we apply the new empirical algorithm oil spread index (OSI) to infer the oil's viscosity.

\subsection{Oil spread index (OSI)}

A fresh oil spill caused by a relatively high viscosity oil should have a continuous edge with either $C_{1}$ or $C_{2}$ curvature type, after applying the sobel filter on it. Fulfilling such conditions means that it is possible to approximate the irregular shape of the spill as a consecutive series of discs whose radii changes in dimension as the spill spreads. Figure 3 shows an example of this, where a circle of radius $r_{1}$ was drawn covering the largest inner area of the spill starting from the spill's assumed source. When drawing the circles, the following should be taken into consideration: (1) the circle perimeter should touch at least two sides of the inner walls of the spill, such that the inner edges of the spill act as tangents to the circle; (2) the circle considered for analysis should have an angle $\Theta$ that lies between the radii of the circle perpendicular to the circle's tangents and whose value should be $100^{\circ}<\Theta<180^{\circ}$; and finally (3) the next adjacent circle with radius $r_{2}$ should be drawn touching the former circle as a tangent. The OSI can then be calculated using equation 2.

2 Non valid data were set to no-value data. 

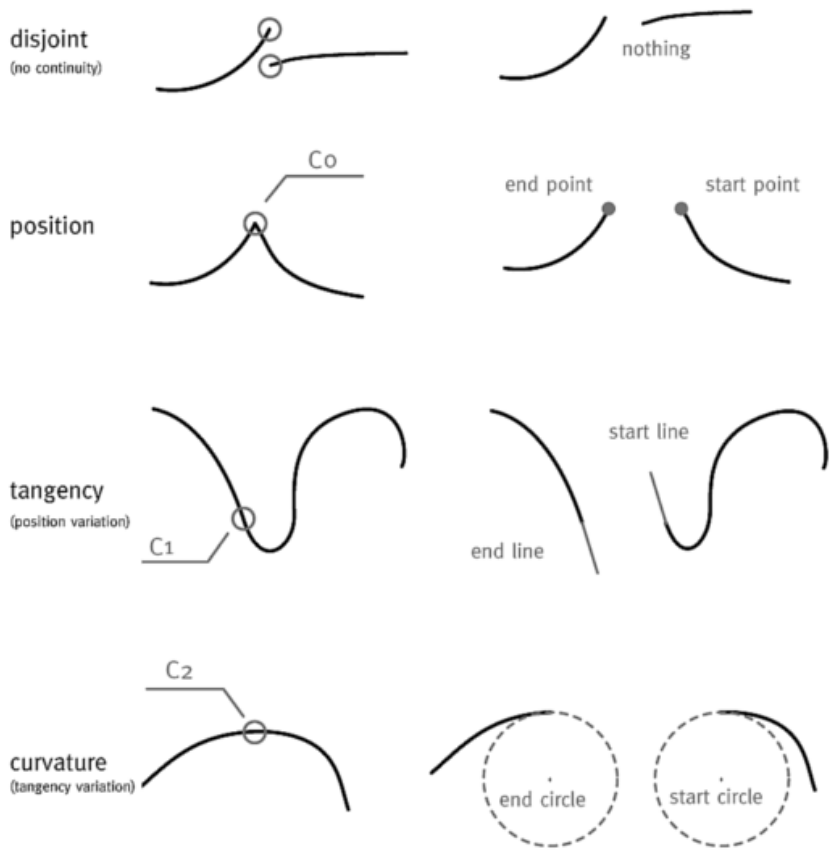

$\mathrm{C}_{2}$ " $\mathrm{C}_{1}$ " $\mathrm{Co}$

Figure 1: $\mathrm{C}_{0}$ continuity - Curves are joined at their endpoints. The connection forms a geometric discontinuity, a sharp edge between surfaces. $C_{1}$ continuity - Connected curves are tangent. The illustration to the right shows the curvature of two lines joined by an arc that is tangent to both lines. $\mathrm{C}_{2}$ continuity - The curvature varies smoothly between connected curves. The illustration to the right shows two lines joined by a spline. Because the curvature of a spline is constantly changing, it can match the zero curvature of the adjacent lines.
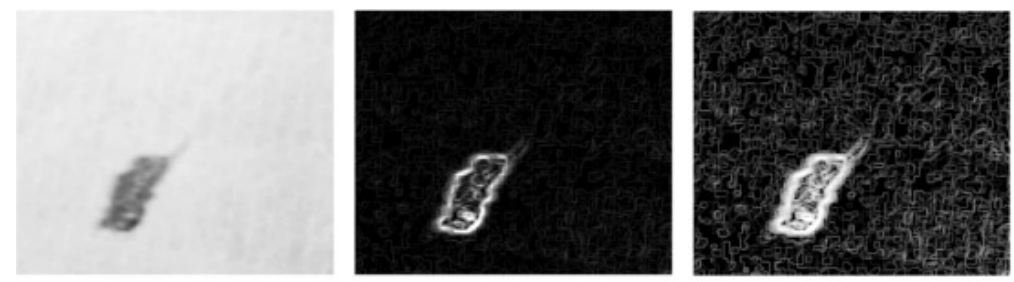

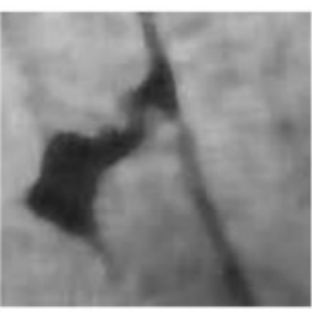

Data

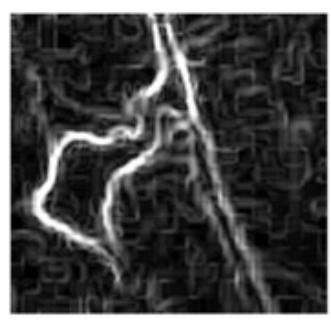

Sobel filter

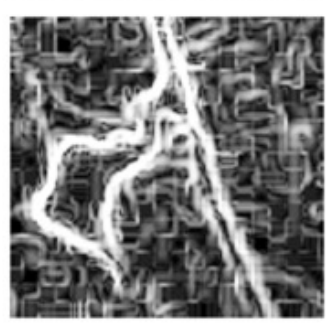

Level stretching

Figure 2: Two images of different oil spills in MODIS data taken on 26 March 09 (top) and Radarsat image taken on 22 March 07 (bottom). The three sets of each image represent: the actual image data; the image after applying the sobel filter; contrast stretching to highlight texture differences respectively. 


$$
O S I=\frac{1}{N} \sum_{i=1}^{i=N} D_{i}
$$

where $\mathrm{N}$ is the number of circles drawn and $\mathrm{D}_{\mathrm{i}}$ is the diameter of the $\mathrm{i}^{\text {th }}$ circle.

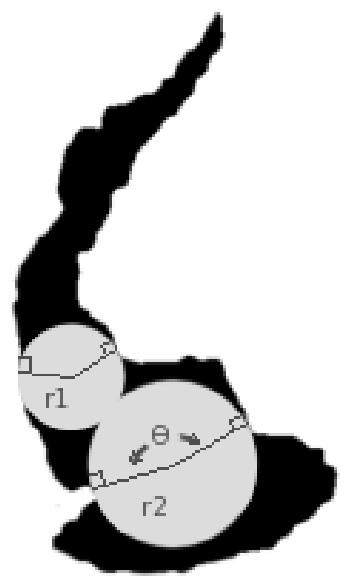

Figure 3: Two circles each with radii r1 and r2 respectively, drawn inside the spill's inner body to demonstrate the methodology for the oil spread index (OSI).

The OSI can be linked to the oil's viscosity ( $\eta$ ) as equation 3 indicates.

$$
\eta \propto \frac{1}{O S I}
$$

This means that if a spill can be represented, for example, with only two large circles (large OSI $\approx$ greater spread) and another spill can be represented also with two circles one of which is larger than the other (small OSI $\approx$ less spread), then the oil of the first spill is less viscous than that in the second spill.

Another alternative interpretation of the term $\sum_{i=1}^{i=N} D_{i}$ can be seen as a representation of the spill's length for that part which is covered by the circles, as Figure 4 shows.

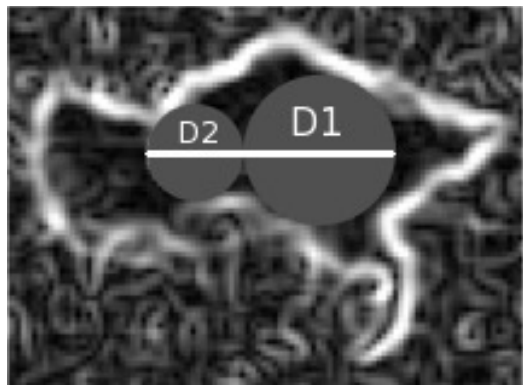

Figure 4: Two circles each with diameter D1 and D2 respectively, drawn inside the spill's inner body to demonstrate the oil spread index (OSI) relation which equals (D1+D2)/2. 


\section{RESULTS}

The analysis carried out for this research involved more than 100 SAR image data and more than 200 of MODIS images representing the optical sensor data. The method outlined was implemented to differentiate between oil and some examples of oil look-alikes substances.

\subsection{Oil look-alikes}

\subsubsection{Floating algae}

Figures 5 and 6 shows two different algae bloom events as they appear in the SAR and MODIS images respectively. The figures also show their corresponding appearance after applying the sobel filter on each of them. In both images algae appears with: (1) no solid uniform bright edges; and (2) discontinuity or with a $\mathrm{C}_{0}$ curvature type, and hence the inability to apply the OSI relation.

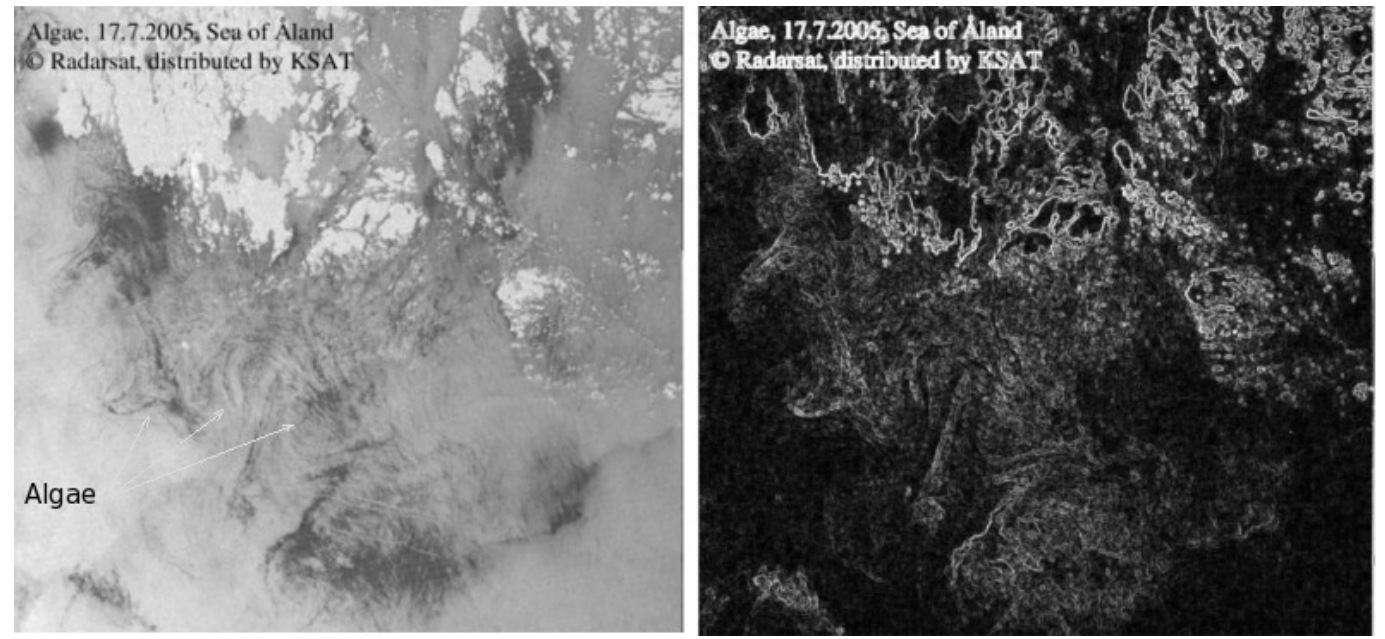

Figure 5: Algae shown in a SAR data (left) and the same image after applying the sobel filter on it (right).

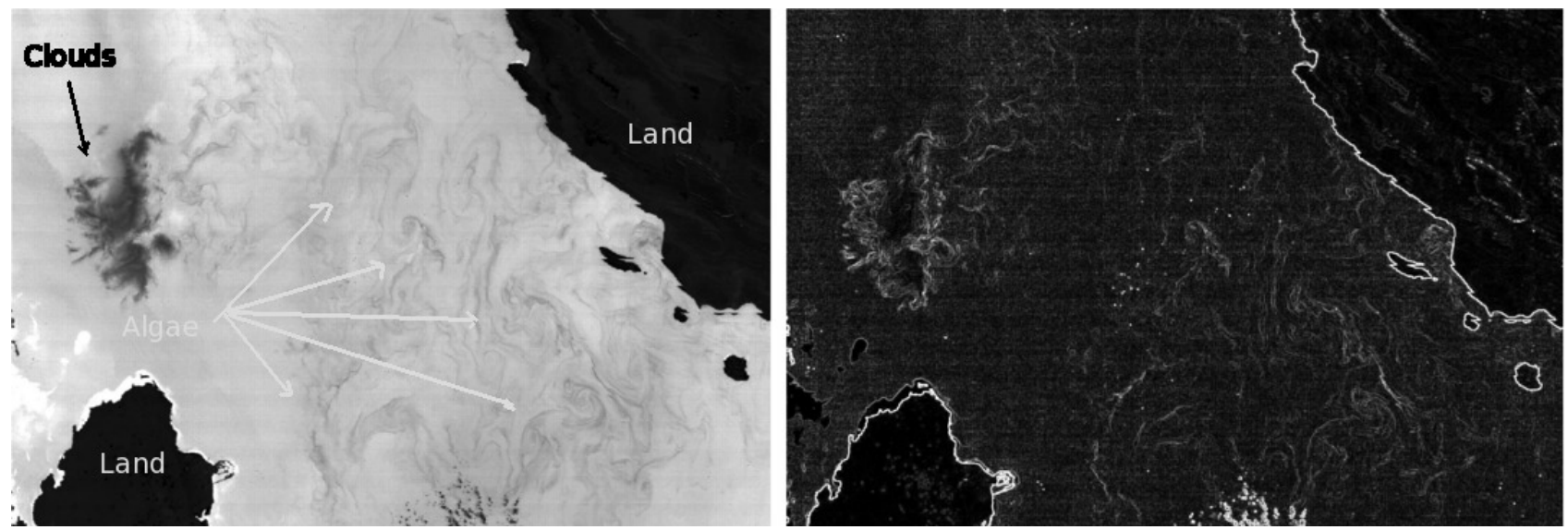

Figure 6: Algae shown in a MODIS data (left) and the same image after applying the sobel filter on it (right). (C) ROPME. 


\subsubsection{Wind patterns}

Figures 7 and 8 shows two different low wind patterns as they appear in the SAR and MODIS images respectively. The figures also show their corresponding appearance after applying the sobel filter on each of them. Wind pattern edges after applying the sobel filter will either be non existent or appearing as tree-like branches with multiple discontinuities or with $\mathrm{C}_{0}$ curvature type. Texturally wind patterns will also appear differently such that their granularity structure compared to those created by the water waves, will almost be non existent in wind patterns; and will therefore appear completely dark even after performing the contrast stretching.
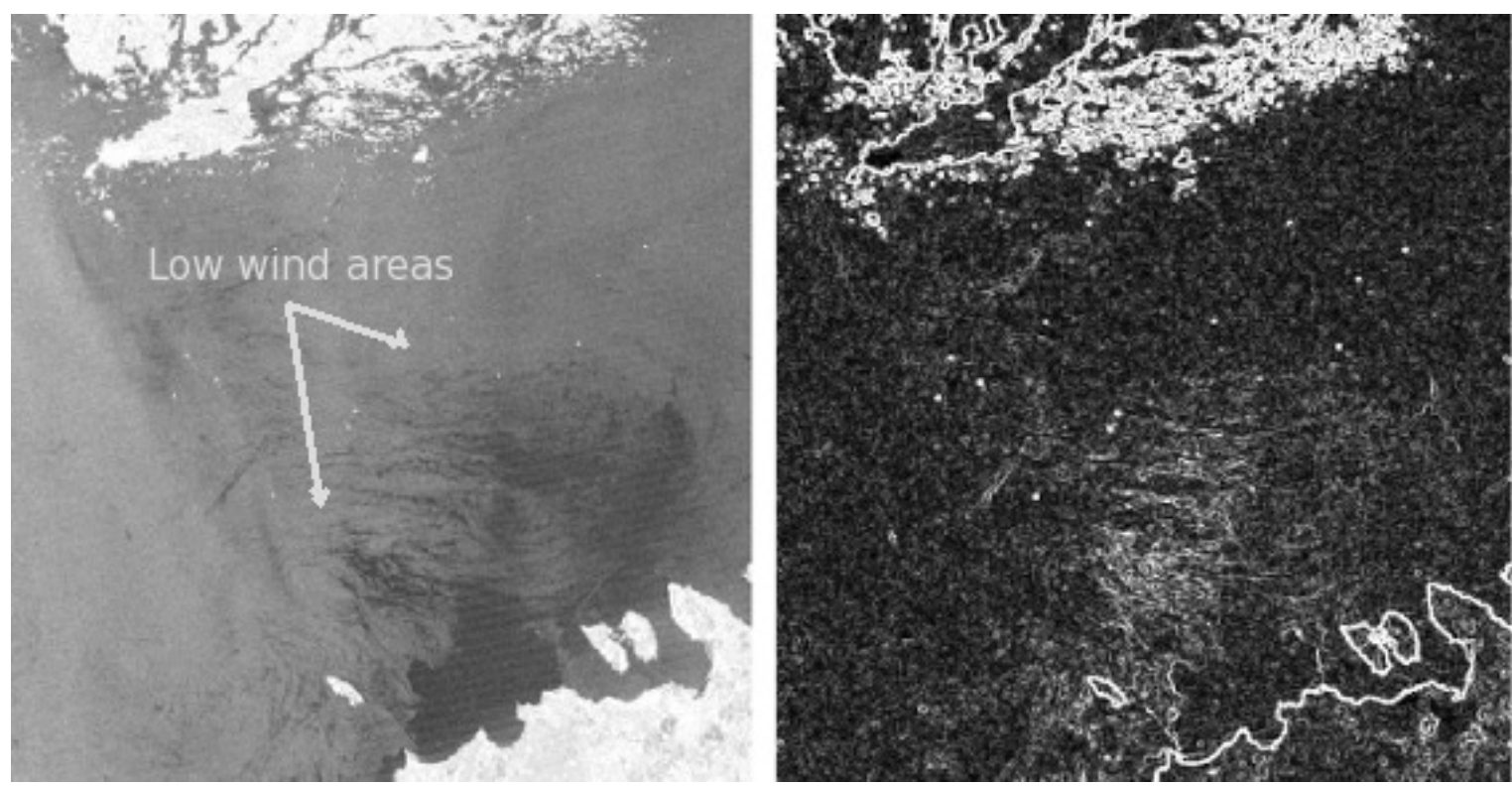

Figure 7: SAR data of low wind areas (left) and the same image after applying the sobel filter on it (right). (C) Canadian Space Agency, distributed by Kongsberg Satellite Service.
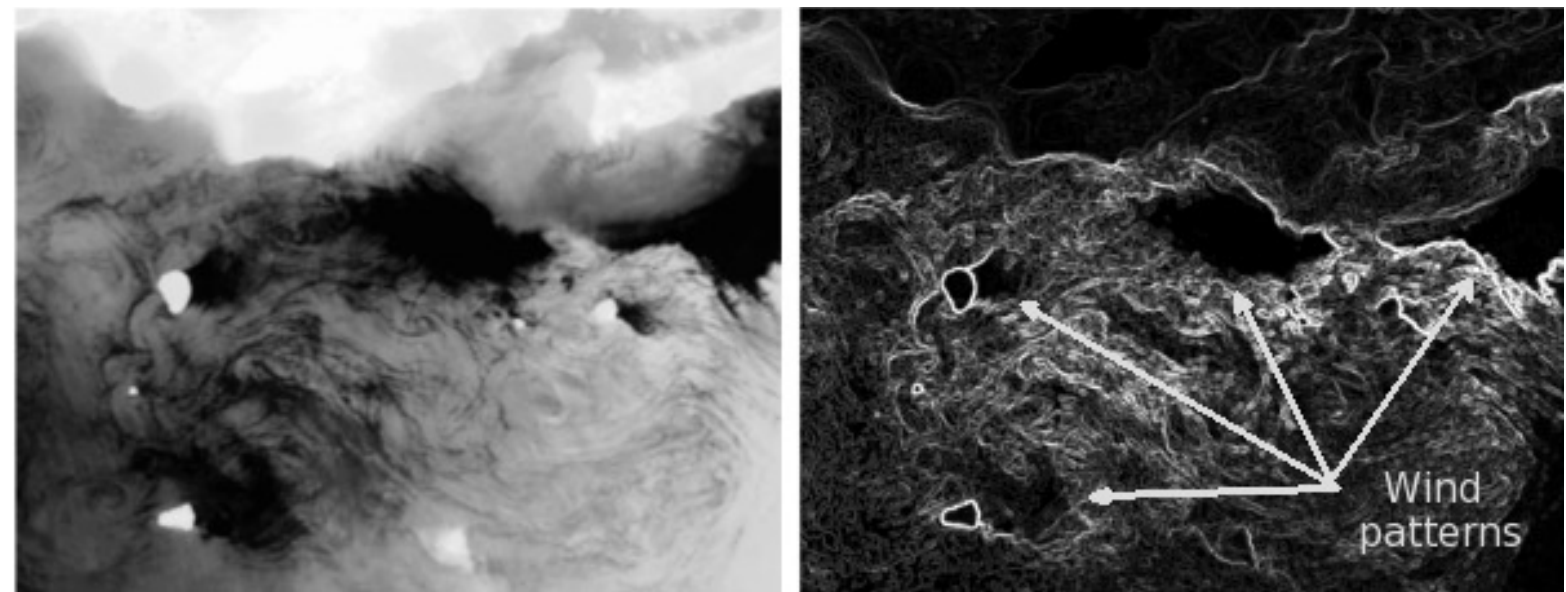

Figure 8: MODIS data of low wind areas (left) and the same image after applying the sobel filter on it (right). (C) ROPME. 


\subsection{Oil spills}

Figure 9 shows a MODIS example of an oil spill and its corresponding image after applying the sobel filter. The spill spatial extent was large enough to apply the OSI method. Because the spilled oil was not pure oil and was instead mixed with other substances, the edges were solid but not uniform in width and were also experiencing slight $\mathrm{C}_{0}$ curvature type away from spill's sources.

Table 2 shows the different OSI values and their corresponding oil viscosity classification found whilst conducting this research.

The spill shown in Figure 9 originated from an offshore oilfield in the RSA. Its oil is classified as of a medium viscosity type, and this matched with the OSI value we have calculated.
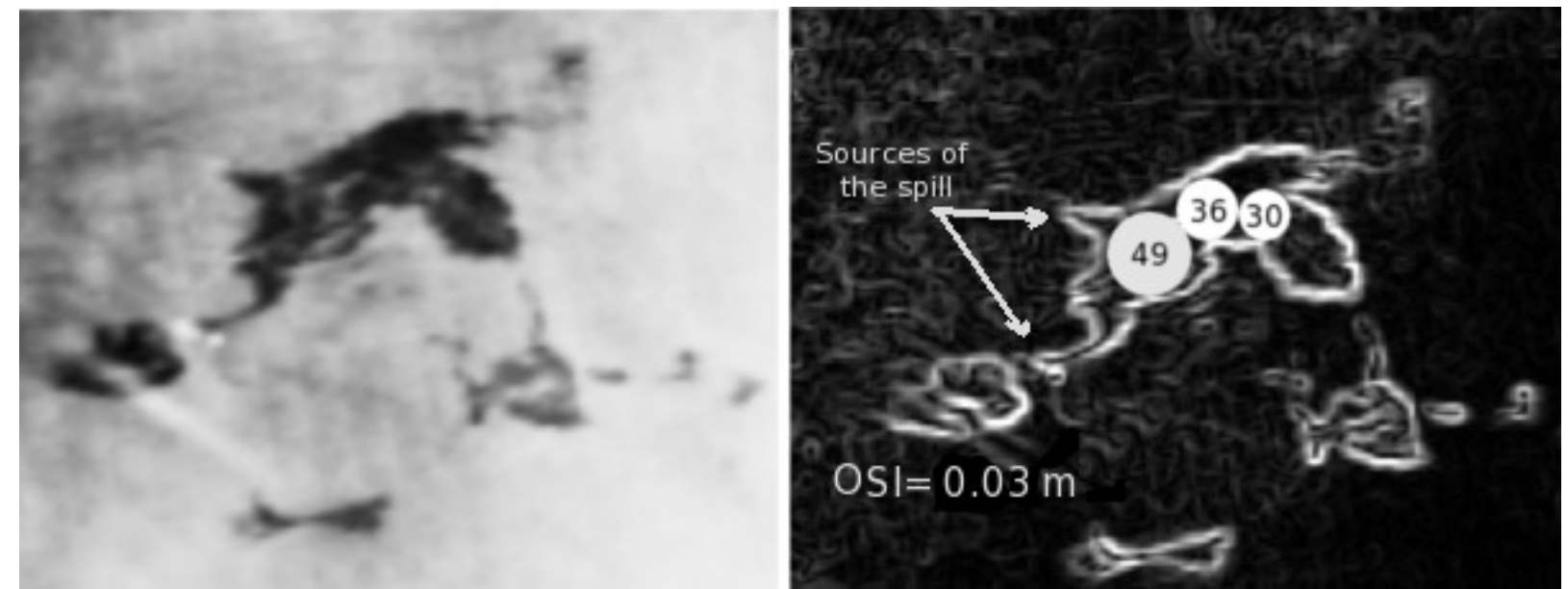

Figure 9: A false colour composite RGB 2, 1, 1 (869nm, 649nm, 649nm) at 250m/pixel spatial resolution of MODIS Aqua taken on 30 June 09 showing an oil spill from an offshore platform in RSA (left) and the same image after applying the sobel filter and the application of the OSI relation (right). (C) ROPME.

\begin{tabular}{|c|c|}
\hline Classification of oil viscosity & OSI \pm 0.005 \\
\hline Light & $0.1-0.05$ \\
\hline Medium & $0.03-0.04$ \\
\hline Heavy & $0.01-0.02$ \\
\hline
\end{tabular}

Table 2: The different OSI values and their corresponding oil viscosity classification.

Figure 10 shows a SAR image taken from ENVISAT of an oil spill that occurred in the Irish waters, together with the corresponding image after applying the sobel filter on it. Because the oil type spilled was light fuel oil, the edges' brightness were not well defined (variable edge width at certain parts) and experiencing slight $\mathrm{C}_{0}$ curvature type away from spill's source. The OSI value came to be 0.04 , which classifies the oil to be of medium type viscosity and not matching the actual oil type involved in the accident. The discrepancy might be have been attributed to the weathering of the oil which lead its viscosity to change resembling a higher viscous oil type. 


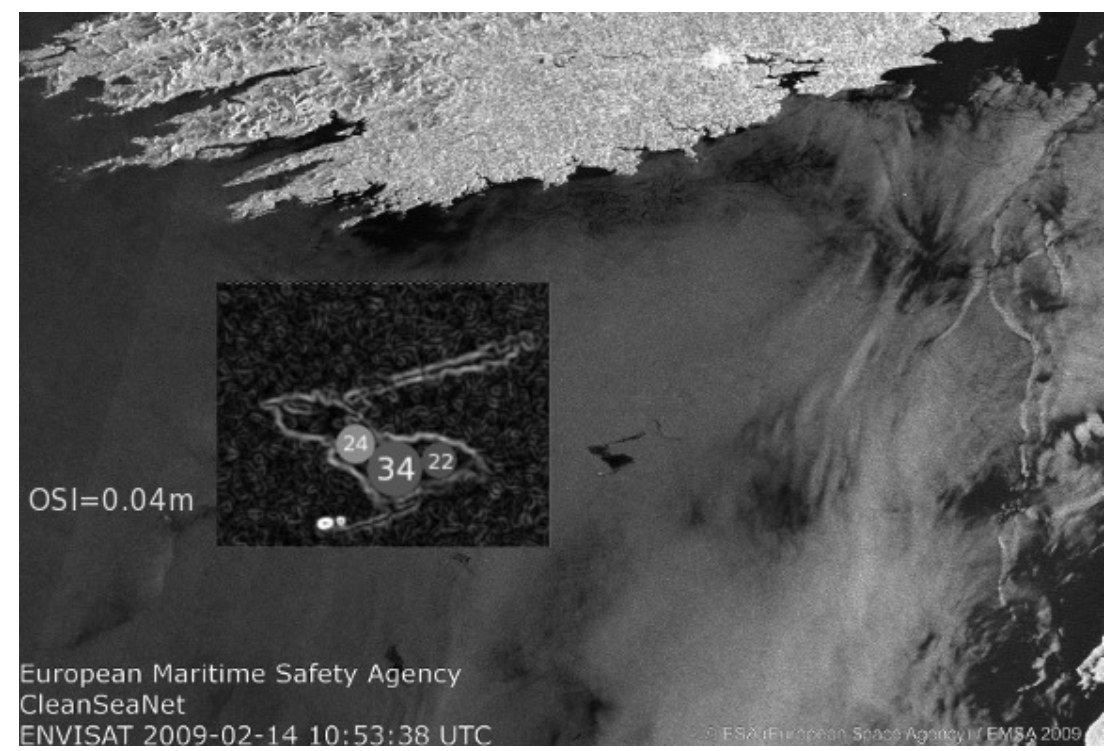

Figure 10: A SAR image showing an oil spill in the Irish waters on 14 February 09 and the same image after applying the sobel filter with the OSI value shown (inset).

Extreme ageing or weathering (non fresh) oil spills dos not make them adhere to the shape classification methods proposed herein. One notable shape example for such case is the 'feathered' shape oil spill shown in Figure 11. Such shapes are created when the wind causes the heavy components of the oil film to accumulate at the downwind side (dark line in the image). The feathered side is always located upwind. The feathering is caused by vortices known as Langmuir circulation which split the spill into streaks [14,19].

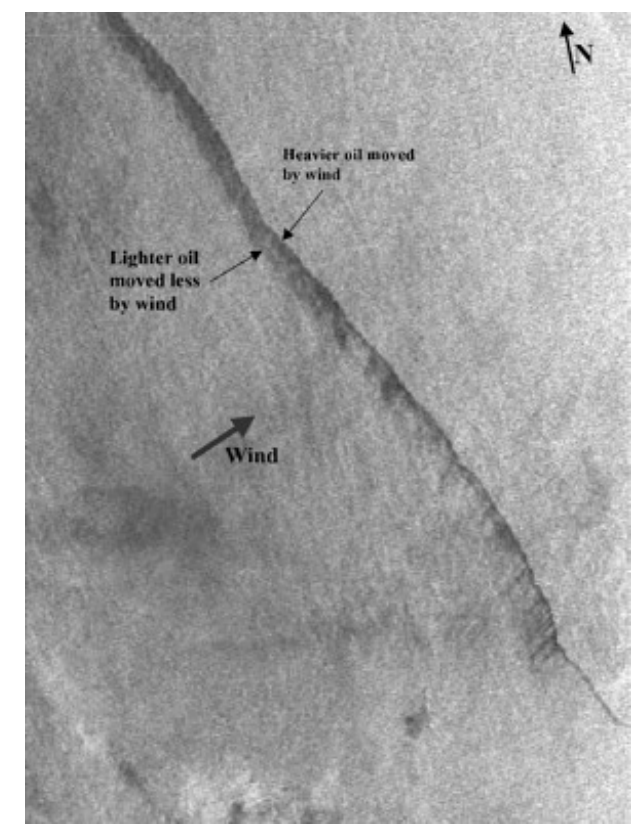

Figure 11: Section of an ERS-2 SAR image from the Indian Ocean acquired 6 April 1999 at 04 showing a "feathered" structure of an oil trail. CESA 1999. 


\section{CONCLUSION}

The aim of this research was to identify a robust method by which it is possible, with the minimal available ancillary data to identify (with high probability) oil spills from the shape they appear in satellite data. The analysis is confined to spatially large fresh oil spills originating from offshore stationary sources. Applying the sobel edge filter on spills captured by satellite data (SAR or optical sensors), will characterize the edges of the spill with uniform brightness; continuity ( $\mathrm{C}_{1}$ or $\mathrm{C}_{2}$ curvature types); and a texture granularity similar to the surrounding sea water surface, but less in texture complexity due to the dampening effect of the viscous oil film. These unique shape and texture features which oil spills exhibit, were used to discriminate between them and other lookalikes.

It has been demonstrated that oil spills that adhere to such shape conditions satisfy the criterion set by the empirical relationship termed oil spread index (OSI). The validity of this method depends on a number of assumptions made: oil viscosity remains constant and consequently the parameters it depends on are also assumed to remain unchanged like temperature, wave height and speed; and the purity of the oil (that is not mixed with other substances). The shapes oil spills adopt depend primarily on their viscosity and the OSI was used to estimate its level. Fresh spills caused by heavy to medium oil types (higher viscosity than water) like crude and heavy crude, will have well defined sobel filtered edges upon which the method proposed herein will be most effective. However, the proposed method will produce inconclusive results with regard to spills created by light to very light oil types, whose viscosity are either close to or less than water viscosity, like gasoline and diesel. Therefore, additional ancillary data will be required to provide a more accurate determination.

In order to apply the proposed techniques, the spill's spatial extent should be large enough to allow for the implementation of the OSI relation.

Rigorous work remains to be carried out to verify the relationship between the oil viscosity and the OSI values, to include the effects of other viscosity related variables like wind-sea states and temperature.

\section{ACKNOWLEDGEMENT}

Special thanks are due to Dr. Peter Petrov for his valuable contribution towards the preparation of this paper. 


\section{REFERENCES}

[1] Alawadi, F., Amos, C., Byfield, V., et al., "The application of hyperspectral image techniques on MODIS data for the detection of oil spills in the RSA,” Proceedings of SPIE Vol. 7110, 71100Q (2008).

[2] Buckmaster, J., “Viscous-gravity spreading of an oil slick” Journal of Fluid Mechanics, 59(3), 481-491 (1973).

[3] Carracedo, P. , Torres-López, S., Barreiro, M. , Montero, P. , Balseiro, C.F. , Penabad, E. , Leitao P.C. , and PérezMuñuzuri V., "Improvement of pollutant drift forecast system applied to the Prestige oil spills in Galicia Coast (NW of Spain): Development of an operational system” Marine Pollution Bulletin 53, 350-360 (2006).

[4] Chust, G. and Sagarminaga, Y., "The multi-angle view of MISR detects oil slicks under sun glitter conditions. Remote Sensing Environment”, 107(1-2): 232-239 (2007).

[5] Dessì, F., Melis, M. T., Naitzaa,L. and Marini, A., "MODIS data processing for coastal and marine environment monitoring: A study on anomaly detection and evolution in gulf of cagliari (sardinia-italy)” ISPRS., XXXVII. Part B8. Commission VIII, WG VIII/6, (2008).

[6] Elliot, A., "Shear diffusion and the spread of oil in the surface layers of the North Sea" German Journal of Hydrography. 39, 113-137 (1986).

[7] Espedal, H. "Detection of oil spill and natural film in the marine environment by spaceborne SAR", Ph.D. thesis, Department of Physics, University of Bergen, (1998).

[8] Fay, J.A., “The spread of oil slicks on a calm sea. In: (eds) Oil on the Sea” Plenum Press, New York, 53-63 (1969).

[9] Fingas, M., and C. Brown, "Oil-spill remote sensing -- an update," Sea Technology. 41, 21-26 (2000).

[10] Hoult, D.P., “Oil Spreading on the Sea” Department of Mechanical Engineering, Massachusetts Institute of Technology. Cambridge, MA, 341-368 (1972).

[11] Hovland, H.A., Johannessen, J.A., Digranes, G., "Slick detection in SAR images" In: Proceedings of the IEEE Symposium on Geoscience and Remote Sensing (IGARSS), Pasadena, CA, 2038-2040 (1994).

[12] Huang, J.C., "Review of the State of the Art of Oil Spill Fate-Behavior Models” Proceedings: Oil Spill Conference, American Petroleum Institute, 313-322 (1983).

[13] Jordan, R.E. and Payne, J.R., "Fate and Weathering of Petroleum Spills in the Marine Environment”. Ann Arbor Science (1980).

[14] Langmuir, I., “Surface motion induced by the wind” Science 87, 119-123 (1938).

[15] Lijian, S., Zhang, X., Seielstad, G., Zhao, C., He, M., “Oil Spill detection by MODIS Images using Fuzzy Cluster and Texture Feature Extraction” Oceans 2007, 1-5 (2005).

[16] Otremba Z. and iskozub.J., "Modelling of the optical contrast of an oil film on a sea surface”. Optics Express, 9, 411-416 (2001).

[17] Otremba Z., "Selected results of light field modelling above the sea surface covered by thin oil film” 41'th issue of series: Computer Simulation and Boundary field problems, Environmental Simulations, Ed.: Riga Technical University, 5-13 (1999). 
[18] Otremba Z., Król T., "Light attenuation parameters of polydisperse oil-in-water emulsion” Optica Applicata, 31(3): 600-609 (2002).

[19] Pavlakis, P., D. Tarchi, and A. J. Sieber, "On the monitoring of illicit vessel discharges using spaceborne SAR remote sensing” A reconnaissance study in the Mediterranean Sea. Ann. Telecommun., 56, 700-718 (2001).

[20] Payne, J.R. and Phillips, C.R., "Petroleum Spills in the Marine Environment”. Lewis Publishing Incorporated (1985).

[21] ROPME, "Kuwait Regional Convention for Cooperation on the Protection of the Marine Marine Environment and the Coastal Areas of Bahrain, Iran, Iraq, Kuwait, Oman, Qatar, Saudi Arabia and United Arab Emirates, Kuwait]" ROPME, 5 (1978).

[22] Solberg, A. H. S., Storvik, G., Solberg, R. and Volden, E., “Automatic detection of oil spills in ERS SAR images” IEEE Transactions on Geoscience and Remote Sensing, 37, 1916-1924 (1999).

[23] Spaulding, M.L., “A state-of-the-art review of oil spill trajectory and fate modeling” Oil Chemical Pollution 41, 3955 (1988).

[24] Stathakis, D.; Topouzelis, K.; Karathanassi, V., "Large-scale feature selection using evolved neural networks" SPIE, Image and Signal Processing for Remote Sensing XII, Bruzzone, Lorenzo Ed.; SPIE Bellingham Wash. ETATSUNIS: Stockholm, Sweden, 6365 (2006).

[25] Tsukihara, T., "Weathering experiment on spilled crude oils using a circulating water channel” Proceedings of the 1995 International Oil Spill Conference, 435-442 (1995).

[26] Valenzuela, G. R., "Theories for the interaction of electromagnetic and ocean waves-A review” Bound.-Layer Meteor 13, 61-85 (1978). 